\title{
Impacto de los pasivos ambientales mineros en la variación de la calidad del agua en la quebrada colorada, setiembre 2015 - agosto 2016
}

\section{Impact of mining environmental liabilities on the variation of water quality in the colorada quebrada, september 2015 - august 2016}

\author{
Eliana M Cabrejos $\mathrm{B}^{1}$., Mirtha Culqui L ${ }^{2}$., Aleida $\mathrm{S}$ Cabrejos $\mathrm{B}^{3}$ y Wilter J Vásquez $\mathrm{M}^{4}$.
}

\begin{abstract}
RESUMEN
En el distrito de Chugur, Hualgayoc, Cajamarca, se explota minerales desde siglo XVIII hasta la actualidad, en este período se acumuló grandes cantidades de relaves y otros pasivos ambientales. El objetivo de la presente investigación fue determinar la variación de la calidad del agua en la quebrada Colorada por efecto de los pasivos ambientales mineros desde setiembre 2015 hasta agosto 2016. Se tomaron muestras en seis periodos diferentes entre setiembre del 2015 y agosto del 2016, considerando: avenida, transición y estiaje propios del ciclo hidrológico, para ello se consideró lo establecido por el Protocolo Nacional de Monitoreo de la Calidad de las Aguas Superficiales, aprobado mediante Resolución Jefatural 182-2011-ANA y Resolución Jefatural 010-2016-ANA. Se obtuvo el análisis de veinticinco parámetros, los mismos que fueron comparados con los Estándares de Calidad Ambiental para Agua, categoría 3 "Riego de vegetales y bebida de animales". Los pasivos ambientales mineros originan la variación de la calidad del agua en el arroyo Colorada cuyo valor de $\mathrm{pH}$ es preponderantemente acido llegando a un valor de 2.90, asimismo los metales aluminio, arsénico, cadmio, cobalto, cobre, hierro, manganeso, plomo y zinc registraron valores que excedieron lo establecido en el ECA - Agua para la categoría 3.
\end{abstract}

Palabras clave: pasivo ambiental, pasivo ambiental minero, calidad del agua.

\begin{abstract}
In the district of Chugur, Hualgayoc, Cajamarca, minerals are exploited from the eighteenth century until today, in this period accumulated large amounts of tailings and other environmental liabilities. The objective of this investigation was to determine the variation in water quality in Colorada Creek due to the effect of mining environmental liabilities from September 2015 to August 2016. Samples were taken in six different periods between September 2015 and August 2016, considering: avenue, transition and sewage typical of the hydrological cycle, for this purpose the provisions established by the National Protocol for Monitoring Surface Water Quality were considered, approved through Headquarters Resolution 182-2011-ANA and Headquarters Resolution 010-2016-ANA. The analysis of twenty-five parameters was obtained, the same ones that were compared with the Environmental Quality Standards for Water, category 3 "Vegetable irrigation and animal drink". The mining environmental liabilities originate the variation of the water quality in the Colorada stream whose $\mathrm{pH}$ value is predominantly acidic reaching a value of 2.90 , also the metals aluminum, arsenic, cadmium, cobalt, copper, iron, manganese, lead and zinc recorded values that exceeded the provisions of the ECA - Water for category 3.
\end{abstract}

Keywords: environmental liability, mining environmental liability, water quality.

\footnotetext{
${ }^{1}$ Universidad Nacional Pedro Ruiz Gallo, Perú. Email: elianacabrejos@gmail.com

${ }^{2}$ Superintendencia Nacional de Agua y Saneamiento - SUNASS. Cajamarca. Email: mirtita07@ hotmail.com

${ }^{3}$ Departamento de Aseguramiento de la Calidad CENCOSUD, Chiclayo. Email: adalei@outlook.como.pe

${ }^{4}$ Consultor de proyectos electromecánicos. Email: javame47@ hotmail.com
} 


\section{INTRODUCCIÓN}

La Ley de Recursos Hídricos № 29338 y su reglamento contribuyen a la modernización de la gestión de los recursos hídricos del país, transformando pautas conductuales y culturales relativas al valor, uso y gestión del agua de los sectores sociales y productivos (minería). Este marco normativo intenta generar el eficiente aprovechamiento, minimizando impactos negativos en los ecosistemas acuáticos, teniendo como ente rector a la Autoridad Nacional del Agua adscrito al Ministerio de Agricultura.

La quebrada Colorada, según el marco normativo actual está considerado como un cuerpo de agua de $10.57 \mathrm{Km}$ de longitud, ubicada en la cuenca Chancay Lambayeque; en la mencionada quebrada existe influencia marcada de catorce (14) pasivos ambientales mineros (socavones con agua y sin agua, canchas de relave, planta en abandono, laguna cleopatra) que afecta la calidad del agua, los mismos que fueron ubicados en el Paraje Sinchao, en el distrito de Chugur, provincia de Hualgayoc, que tienen influencia directa en la calidad del agua en la quebrada Colorada; con respecto a la calidad físico química del agua en la quebrada colorada se puede afirmar que el valor de $\mathrm{pH}$ es preponderantemente acido llegando a un valor de 2.90 asimismo los metales aluminio, arsénico, cadmio, cobalto, cobre, hierro, manganeso, plomo y zinc registraron valores que excedieron lo establecido en el ECA - Agua para la categoría 3.

En este contexto, el estudio realizado generará un mejor y nuevo conocimiento científico que permita tomar decisiones bajo el paradigma del desarrollo sostenible con responsabilidad social, económica y ambiental.

\section{MATERIALES Y MÉTODOS}

\section{Población y Muestra}

La población en la presente investigación está determinada por el agua que discurren en la quebrada Colorada, ubicada en el distrito de Chugur, provincia de Hualgayoc, departamento de Cajamarca. La muestra está constituida por los puntos de monitoreo establecidos en la quebrada Colorada: QColo1 aguas abajo a 500 metros, QColo2 a 1000 metros del punto QColo1 y QColo3 a 5000 metros del punto QColo2, según el Protocolo Nacional de Monitoreo de la Calidad de las Aguas Superficiales.

\section{Determinación de la red de monitoreo}

Para la determinación de los puntos de toma de muestra en la quebrada Colorada se ubicaron en zonas donde el cuerpo natural de agua presentó un cauce natural y uniforme, se utilizó el "Protocolo Nacional de Monitoreo de la Calidad de los Cuerpos Naturales de Agua Superficial”, aprobado con Resolución Jefatural 182-2011- ANA y Resolución Jefatural 010-2016- ANA. 
La red de monitoreo, se estableció siguiendo un muestreo no probabilístico por conveniencia de 03 puntos de monitoreo considerando su descripción, coordenadas y altura. El punto de monitoreo se ha identificado de manera que permite su ubicación exacta antes de la toma de muestra de agua. En la determinación de la ubicación se utilizó el Sistema de Posicionamiento Global (GPS); las coordenadas del punto de monitoreo han sido expuestas en sistema UTM en estándar geodésico WGS84, quedando establecidos de la siguiente manera:

Punto de monitoreo 1: QColo1, ubicado en la Quebrada Colorada, a 500 m aguas abajo de los pasivos ambientales, a una altura estimada de 3758 msnm, con coordenadas UTM-WGS84 de 758048 al este y 9257031 al norte.

Punto de monitoreo 2: QColo2, ubicado en la Quebrada Colorada, a 10m del puente, carretera a Chota en $\mathrm{Km} 39$, aguas abajo de los pasivos ambientales de Sinchao, y a 1000m del punto de monitoreo QColo1; a una altura estimada de 3756 msnm, con coordenadas UTM-WGS84 de 756307 al este y 9258607 al norte.

Punto de monitoreo 3: QColo2, ubicado en la Quebrada Colorada - río Perlamayo, 50m aguas arriba de la confluencia con la quebrada Putic y puente peatonal; a una altura estimada de $2653 \mathrm{msnm}$, con coordenadas UTM-WGS84 de 751538 al este y 9262520 al norte.

\section{Determinación de los parámetros físico-químico de las muestras de agua}

Se ha tomado muestras puntuales representativas con un volumen apropiado $(500 \mathrm{ml})$ para analizar los parámetros establecidos, lo cual representa la composición del cuerpo natural de agua superficial, tiempo y circunstancia que fue recolectada la muestra. Los parámetros físico químicos evaluados en la quebrada Colorada son: $\mathrm{pH}$, Temperatura $(\mathrm{T})$, Oxígeno disuelto $\left(\mathrm{O}_{2}\right)$, Sólidos Suspendidos Totales (SST), Conductividad (Cond.), Demanda Química de Oxígeno, Calcio (Ca), Magnesio (Mg), Potasio (K), Sodio (Na ), Aluminio (Al), Antimonio (Sb), Arsénico (As), Bario (Ba), Berilio (Be), Boro (B), Cadmio (Cd), Cobalto (Co), Cobre (Cu), Hierro (Fe), Litio (Li), Manganeso (Mn), Mercurio (Hg), Plomo $(\mathrm{Pb})$, Zinc $(\mathrm{Zn})$.

Los análisis de las aguas para la determinación de parámetros físico químicos se realizaron según los métodos de ensayo acreditados, de acuerdo a los procedimientos de análisis que indica la EPA, APHA-AWWA-WEF, cumpliendo los controles que garantizan a calidad de los resultados. 
Tabla 1. Valores de parámetros analizados en los puntos de monitoreo en la quebrada Colorada

\begin{tabular}{|c|c|c|c|c|c|c|c|c|c|c|c|c|c|c|c|c|c|c|c|c|}
\hline \multirow{2}{*}{$\begin{array}{l}\text { Punto Código de } \\
\text { Parámetro }\end{array}$} & \multirow[t]{2}{*}{ Unidad } & \multirow[t]{2}{*}{$\begin{array}{l}\text { ECA- } \\
\text { Cat.3 }\end{array}$} & \multicolumn{6}{|c|}{ QColo1 } & \multicolumn{6}{|c|}{ QColo2 } & \multicolumn{6}{|c|}{ QColo3 } \\
\hline & & & $\begin{array}{l}\text { Set - } \\
2015 \\
\end{array}$ & Oct_2015 & Dic2015 & $\begin{array}{l}\text { Mar- } \\
2016 \\
\end{array}$ & Jul-2016 & $\begin{array}{l}\text { Agos- } \\
2016 \\
\end{array}$ & $\begin{array}{l}\text { Set - } \\
2015\end{array}$ & Oct_2015 & Dic2015 & $\begin{array}{l}\text { Mar- } \\
2016 \\
\end{array}$ & Jul-2016 & $\begin{array}{l}\text { Agos- } \\
2016 \\
\end{array}$ & $\begin{array}{l}\text { Set - } \\
2015 \\
\end{array}$ & Oct_2015 & Dic2015 & $\begin{array}{l}\text { Mar- } \\
2016 \\
\end{array}$ & Jul-2016 & $\begin{array}{l}\text { Agos- } \\
2016 \\
\end{array}$ \\
\hline $\mathrm{pH}$ & - & $\begin{array}{l}6.5- \\
8.5\end{array}$ & 2.95 & 3.47 & 3.47 & 3.45 & 2.90 & 3.10 & 3.14 & 3.78 & 3.91 & 3.75 & 3.03 & 3.41 & 6.62 & 6.79 & 6.52 & 6.70 & 6.50 & 6.59 \\
\hline Temperatura (T) & ${ }^{\circ} \mathrm{C}$ & |---- & 19.2 & 11.6 & 10.3 & 12.6 & 11.15 & 9.05 & 11.3 & 10.1 & 10.3 & 12.2 & 17.0 & 22.1 & 11.7 & 13.8 & 14.1 & 13.5 & 11.0 & 11.2 \\
\hline $\begin{array}{l}\text { Oxígeno disuelto } \\
\left(\mathrm{O}_{2}\right)\end{array}$ & $\mathrm{mg} / \mathrm{L}$ & $>=4$ & -- & 6.82 & 7.14 & 6.35 & 6.77 & 4.19 & 7.12 & 7.33 & 7.47 & 6.80 & 6.66 & 6.62 & 7.95 & 7.73 & 7.46 & 7.34 & 7.90 & 7.70 \\
\hline $\begin{array}{l}\text { Sólidos } \\
\text { Suspendidos } \\
\text { Totales (SST) }\end{array}$ & $\mathrm{mg} / \mathrm{L}$ & ---- & 50 & 53 & 54 & 59 & 56 & 191.80 & 3 & 5 & 5 & 13 & 3 & 3 & 3 & 4 & 4 & 10 & 3 & 4 \\
\hline $\begin{array}{l}\text { Conductividad } \\
\text { (Cond.) }\end{array}$ & $\mu \mathrm{S} / \mathrm{cm}$ & 2000 & 1719 & 1590 & 1173 & 1034 & 1575 & 1738.00 & 518 & 485 & 402 & 332 & 462 & 571 & 227 & 188.8 & 156 & 260 & 225 & 226 \\
\hline $\begin{array}{l}\text { Demanda Química } \\
\text { de Oxígeno }\end{array}$ & $\begin{array}{c}\mathrm{mg} / \mathrm{L} \\
\mathrm{O} 2 \\
\end{array}$ & 40 & 9.2 & 21 & 24 & $<9$ & 71.71000 & 11.20 & 2.4 & 14 & 43 & $<9$ & $<9$ & $<9$ & 12 & 40 & $<9$ & 10 & 11 & 10 \\
\hline Calcio total (Ca tot) & $\mathrm{mg} / \mathrm{L}$ & 200 & 241.273 & 166.1 & 165.425 & 126.05 & 148.96552 & \begin{tabular}{|l|}
229.0400 \\
\end{tabular} & \begin{tabular}{|l|}
44.9054 \\
\end{tabular} & 40.98 & 36.666 & 34.811 & 29.771 & 39.599 & 32.613 & 29.705 & 26.791 & 29.67 & 31.5 & 30.5 \\
\hline $\begin{array}{l}\text { Magnesio total (Mg } \\
\text { tot) }\end{array}$ & $\mathrm{mg} / \mathrm{L}$ & 150 & 9.9715 & 7.91 & 10.69 & 6.714 & 6.60382 & 8.9100 & 2.0077 & 1.682 & 1.712 & 1.894 & 1.492 & 1.788 & 1.192 & 1.512 & 1.432 & 1.5 & 1.534 & 1.512 \\
\hline Potasio total (K tot) & $\mathrm{mg} / \mathrm{L}$ & ---- & 1.1214 & 1.1 & & 0.9 & 089214 & 1.6900 & 0.924 & & $<0.6$ & $<0.6$ & 1.5 & $<0.6$ & 1 & 08 & 1 & 2 & 1 & 1 \\
\hline Sodio total (Na tot) & $\mathrm{mg} / \mathrm{L}$ & 200 & 1.2575 & & 1.89 & 1.3 & 1.18127 & 1.7300 & 1.9996 & 1.67 & 1.65 & 1.55 & 2.11 & 1.67 & 4.31 & & 3.85 & 4.5 & 4.23 & 4.12 \\
\hline $\begin{array}{l}\text { Aluminio total (Al } \\
\text { tot) }\end{array}$ & $\mathrm{mg} / \mathrm{L}$ & 5 & 12.142 & 18.98 & 40.83 & 14.88 & 14.26439 & 26.2300 & 6.6673 & 5.76 & 5.61 & 3.83 & 9.61 & 5.69 & 1.02 & 4.04 & 1.16 & 1.8 & 1.01 & 1.3 \\
\hline $\begin{array}{l}\text { Antimonio total (Sb } \\
\text { tot) }\end{array}$ & $\mathrm{mg} / \mathrm{L}$ & ---- & 0.002 & 0.003 & 0.28 & 0.0072 & 0.00145 & 0.0090 & $<0.0002$ & $<0,0025$ & 0.0042 & $<0,0025$ & $<0.0025$ & $<0.0025$ & $<0,0025$ & $<0.0025$ & $<0,0025$ & $<0,0025$ & $<0,0025$ & $<0,0025$ \\
\hline $\begin{array}{l}\text { Arsénico total (As } \\
\text { tot) }\end{array}$ & $\mathrm{mg} / \mathrm{L}$ & 0.05 & 0.1227 & 0.254 & 3.182 & 0.172 & 0.01783 & 0.3630 & 0.0052 & 0.006 & 0.097 & 0.019 & $<0.003$ & 0.025 & $<0,003$ & 0.023 & $<0.003$ & $<0.003$ & $<0,003$ & $<0,003$ \\
\hline Bario total (Ba tot) & $\mathrm{mg} / \mathrm{L}$ & 0.7 & 0.0146 & 0.013 & 0.352 & 0.028 & 0.01415 & 0.0290 & 0.0168 & 0.013 & 0.015 & 0.015 & 0.023 & 0.021 & 0.029 & 0.028 & 0.028 & 0.029 & 0.029 & 0.028 \\
\hline $\begin{array}{l}\text { Berilio total (Be } \\
\text { tot) }\end{array}$ & $\mathrm{mg} / \mathrm{L}$ & 0.1 & $<0.0006$ & $8 \mathrm{E}-04$ & 0.0014 & 0.0004 & $<0.001$ & 0.0010 & $<0.0006$ & $<0,0003$ & $<0.0003$ & $<0.0003$ & $<0.0003$ & $<0.0003$ & $<0,0003$ & $<0.0003$ & $<0.0003$ & $<0.0003$ & $<0,0003$ & $<0,0003$ \\
\hline Boro total (B tot) & $\mathrm{mg} / \mathrm{L}$ & 5 & 0.007 & $<0,03$ & $<0.03$ & $<0.03$ & 0.00581 & $<0.002$ & 0.0025 & $<0,03$ & $<0.03$ & $<0.03$ & $<0.03$ & $<0.03$ & $<0,03$ & $<0.03$ & $<0.03$ & $<0.03$ & $<0,03$ & $<0,03$ \\
\hline $\begin{array}{l}\text { Cadmio total (Cd } \\
\text { tot) }\end{array}$ & $\mathrm{mg} / \mathrm{L}$ & 0.005 & 0.1709 & 0.115 & 0.0693 & 0.0773 & 0.02398 & 0.1675 & 0.0214 & 0.0165 & 0.013 & 0.0141 & 0.009 & 0.0196 & 0.0036 & 0.0054 & 0.003 & 0.004 & 0.004 & 0.0045 \\
\hline $\begin{array}{l}\text { Cobalto total (Co } \\
\text { tot) }\end{array}$ & $\mathrm{mg} / \mathrm{L}$ & 0.05 & 0.0646 & 0.065 & 0.0566 & 0.04564 & 0.05676 & 0.0882 & 0.0143 & 0.01265 & 0.01265 & 0.01041 & 0.0111 & 0.01528 & 0.00296 & 0.00535 & 0.00278 & 0.003 & 0.0032 & 0.0032 \\
\hline Cobre total $(\mathrm{Cu}$ tot $)$ & $\mathrm{mg} / \mathrm{L}$ & 0.2 & 22.9916 & 17.06 & 14.729 & 15 & 3.98528 & 20.9990 & 3.2715 & 2.734 & 2.422 & 2.43 & 2.179 & 3.69 & 0.408 & 1.227 & 0.44 & 0.46 & 0.41 & 0.41 \\
\hline Hierro total (Fe tot) & $\mathrm{mg} / \mathrm{L}$ & 1 & & 74.76 & 198.243 & 102.304 & 59.49703 & 145.5000 & & 5.539 & 11.796 & 10.315 & 2.641 & 9.026 & & 4.021 & 1.514 & 1.6 & 0.151 & 0.151 \\
\hline Litio total (Li tot) & $\mathrm{mg} / \mathrm{L}$ & 2.5 & 0.0084 & 0.008 & 0.0146 & 0.0036 & 0.00574 & 0.0110 & 0.0033 & 0.0027 & $<0.0027$ & $<0.0027$ & 0.0036 & $<0.0027$ & $<0,0027$ & $<0.0027$ & $<0.0027$ & $<0.0027$ & \begin{tabular}{|l|}
$<0,0027$ \\
\end{tabular} & $<0,0027$ \\
\hline $\begin{array}{l}\text { Manganeso total } \\
\text { (Mn tot) }\end{array}$ & $\mathrm{mg} / \mathrm{L}$ & 0.2 & 21.9687 & 19.81 & 9.322 & 10.6658 & 15.14819 & 19.5929 & 3.1664 & 3.1577 & 2.352 & 2.113 & 1.5238 & 3.1174 & 0.6146 & 0.9855 & 0.5169 & 0.413 & 0.595 & 0.6 \\
\hline $\begin{array}{l}\text { Mercurio total }(\mathrm{Hg} \\
\text { tot) }\end{array}$ & $\mathrm{mg} / \mathrm{L}$ & 0.001 & $<0.0001$ & $<0,0003$ & 0.0023 & $<0.0001$ & 0.00000 & 0.0000 & $<0.0001$ & $<0,0003$ & $<0.0003$ & $<0.0001$ & $<0.00010$ & $<0.0001$ & $<0,0003$ & $<0.0003$ & $<0.0001$ & $<0.0001$ & $<0,0003$ & $<0,0003$ \\
\hline Plomo total ( $\mathrm{Pb}$ tot) & $\mathrm{mg} / \mathrm{L}$ & 0.05 & 0.0377 & 0.057 & 0.724 & 0.0579 & 0.03838 & 0.0716 & 0.0235 & 0.02 & 0.023 & 0.0139 & 0.0292 & 0.0263 & $<0,003$ & 0.01 & $<0.001$ & $<0.001$ & $<0,002$ & $<0,002$ \\
\hline Zinc total (Zn tot) & $\mathrm{mg} / \mathrm{L}$ & 2 & 28.5498 & 17.01 & 10.386 & 9.691 & 3.48013 & 21.5970 & 3.4744 & 2.623 & 1.886 & 2.152 & 1.336 & 3.09 & 0.623 & 0.821 & 0.421 & 0.421 & 0.587 & 0.587 \\
\hline
\end{tabular}




\section{Técnica de procesamiento de y análisis de datos}

En la técnica de procesamiento ha sido necesario considerar la red de monitoreo. Para el análisis de los datos, estos han sido registrados en los meses de setiembre, octubre, diciembre del año 2015, marzo, julio y agosto del 2016, analizándose los promedios de la presencia de los parámetros físico químicos en los tres puntos de monitoreo por la influencia de los pasivos ambientales mineros ubicados en el ámbito de la quebrada Colorada; con una probabilidad de $\mathrm{p}<0.05$, se empleó la prueba de Tuckey, para determinar la variación de la calidad físico química del agua en la quebrada Colorada. Los análisis estadísticos de los datos se procesaron empleando los softwares Excel, Google Earth y Spss 23. Las pruebas estadísticas aplicadas fueron el análisis de varianza (ANAVA) y la prueba de comparaciones múltiples (Tukey): El Análisis de Varianza (ANAVA) comparó las características de calidad en puntos de monitoreo y entre periodos de muestreo todos a la vez. La prueba de comparación múltiples (Tukey) comparó los parámetros por cada punto de monito: QColo1, QColo2 y QColo3; a fin de examinar exactamente entre cuáles de ellos había variación estadística. Las pruebas estadísticas fueron realizadas empleando un nivel de significancia del $5 \%$, de manera que si $\mathrm{p}<0.05$ indica que hay diferencia estadística.

\section{RESULTADOS}

Se realizó el análisis de veinticinco (25) parámetros, ver Tabla 1.

El promedio del $\mathrm{pH}$ es diferente en los tres puntos de monitoreo. $(\mathrm{p}=0.000<0.005)$. En cuanto al $\mathrm{pH}$ las diferencias son más notorias en el punto de monitoreo QColo3. Respecto a los valores de pH (unidad de $\mathrm{pH}$ ) en la quebrada Colorada varió desde 2.95 en la muestra de agua tomada en el punto de monitoreo QColo1, en setiembre 2015, hasta 6.79 en el QColo3, (Tabla 1 y Figura 1.) y varió desde 2.9 en la muestra de agua tomada en el punto de monitoreo QColo1, en julio 2016, hasta 6.7 en el QColo3, en marzo 2016 (Tabla 1 y Figura 2.). 


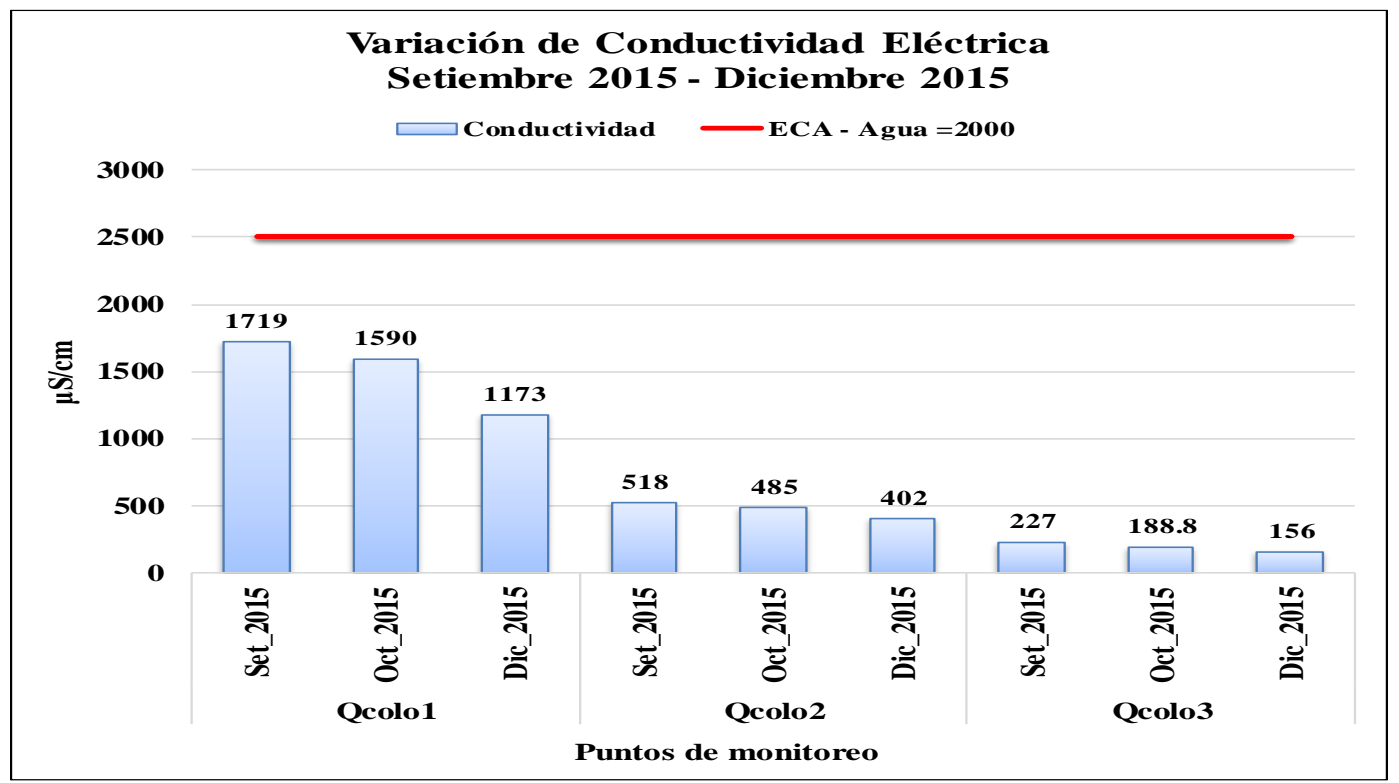

Figura 1. Variación de la conductividad eléctrica en la calidad del agua de la quebrada Colorada, setiembre - diciembre 2016. Según DS N002-2008 MINAN

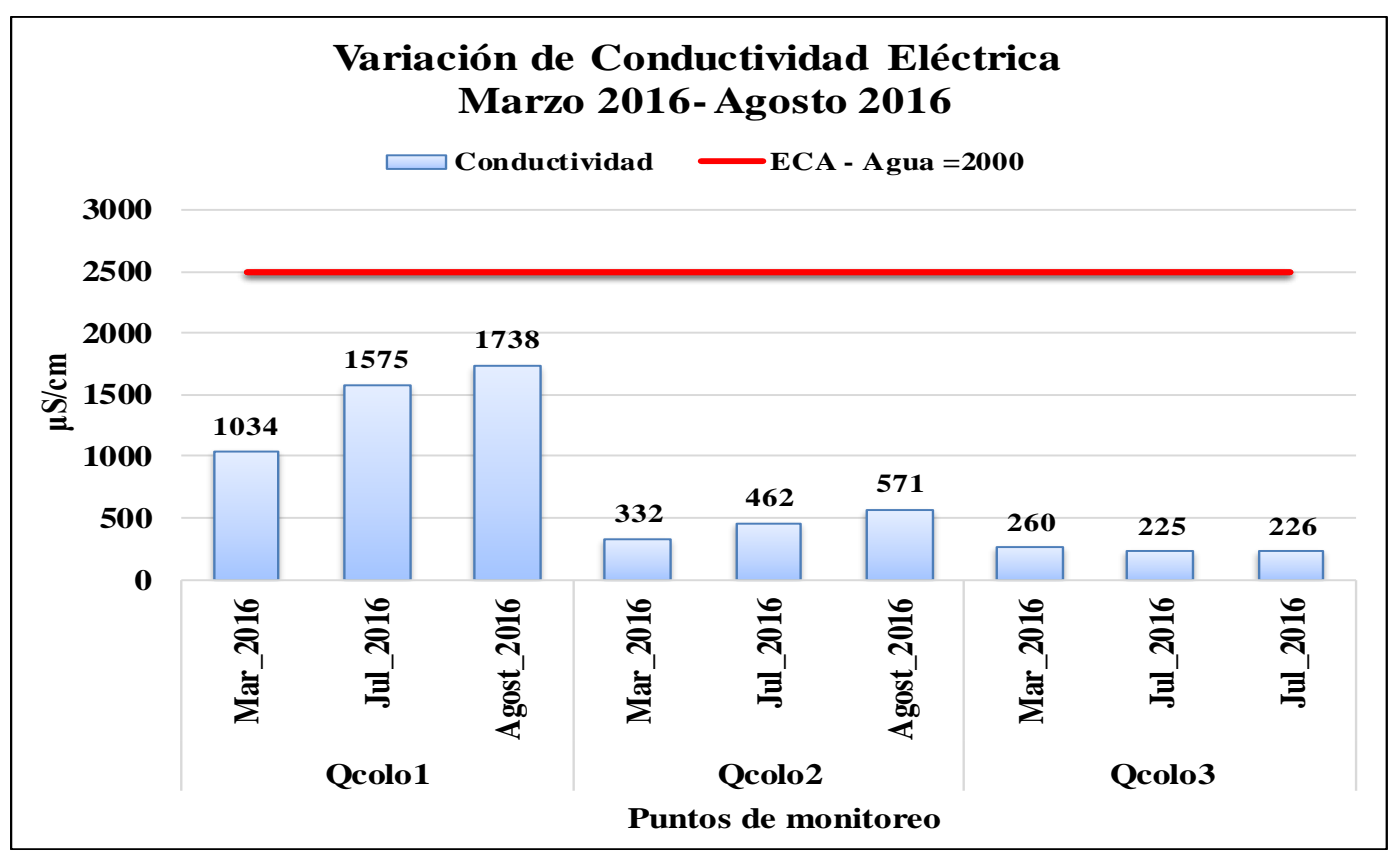

Figura 2. Variación de la conductividad eléctrica en la calidad del agua de la quebrada Colorada, marzo - agosto 2016. DS N015-2015 MINAN

$\mathrm{El}$ promedio del $\mathrm{Al}$ es diferente en los tres puntos de monitoreo. $(\mathrm{p}=0.000<0.005)$. En cuanto al $\mathrm{Al}$ las diferencias son más notorias en el punto de monitoreo QColo1. 
Los valores de aluminio (mg/l) en la quebrada Colorada varió desde 1.02 en la muestra de agua tomada en el punto de monitoreo QColo3, en setiembre del 2015 hasta 40.83 en el QColo1 en diciembre del 2015 (Tabla 1. y Figura 3.); y varió desde 1.01 en la muestra tomada en el punto de monitoreo QColo3 en Julio del 2016 hasta 26.23 en el QColo1 en agosto del 2016 (Tabla 1. y Figura 4.).

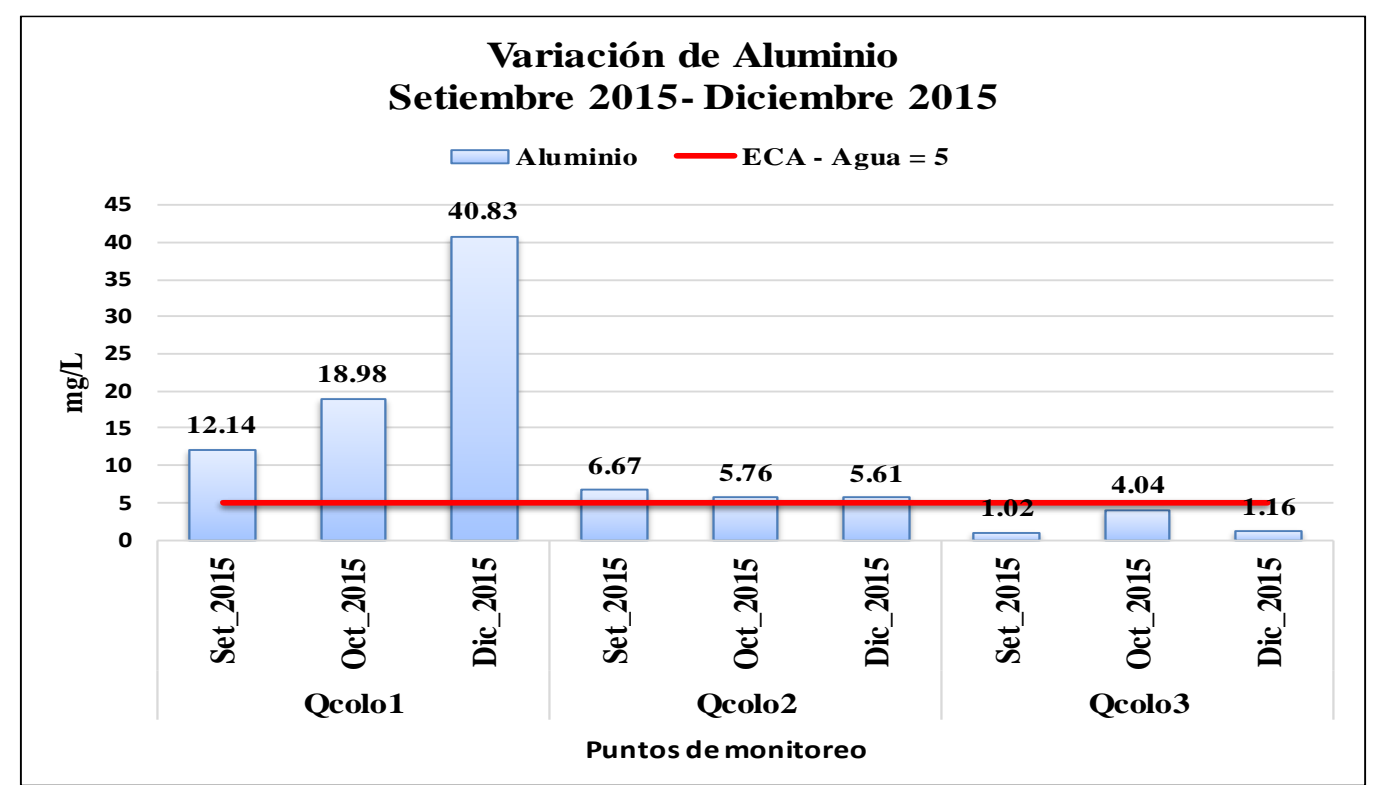

Figura 3. Variación de aluminio en la calidad del agua de la quebrada Colorada, setiembre - diciembre 2015. Según DS $\mathrm{N}^{\circ} 002-2008$ MINAN

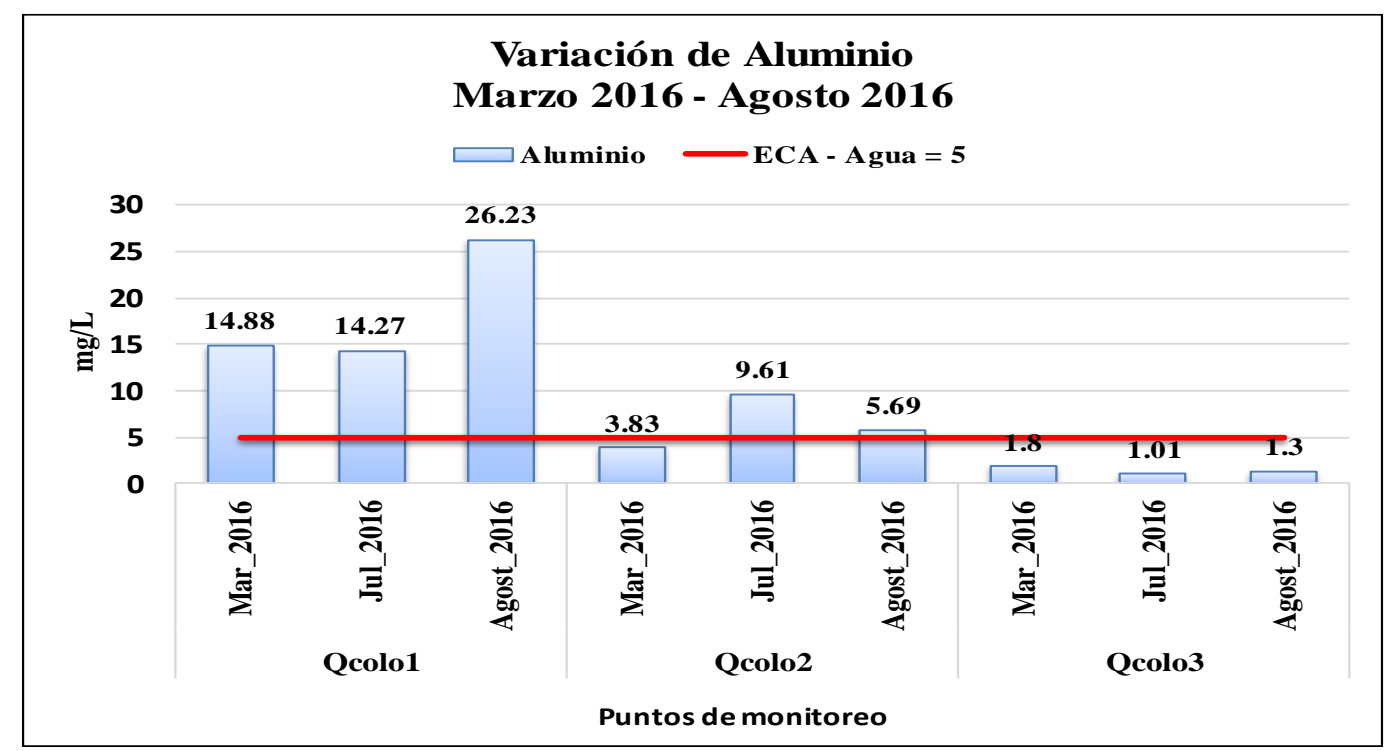

Figura 4. Variación de Aluminio en la calidad del agua de la quebrada Colorada, Marzo - Agosto 2016. Según DS

$$
\mathrm{N}^{\circ} 015-2015 \text { MINAN }
$$

El promedio del As se mantiene en los tres puntos de monitoreo. $(\mathrm{p}=0.203>0.005)$. Los valores de arsénico (mg/l) en la quebrada Colorada varió desde 0.00 en la muestra de agua tomada en el punto de monitoreo QColo3, en setiembre del 2015 hasta 3.18 en el QColo1 en diciembre del 2015 (Tabla 
1 y Figura 5); y varió desde 0.00 en la muestra tomada en el punto de monitoreo QColo2 en julio del 2016 hasta 0.36 en el QColo1 en agosto del 2016 (Figura 6).

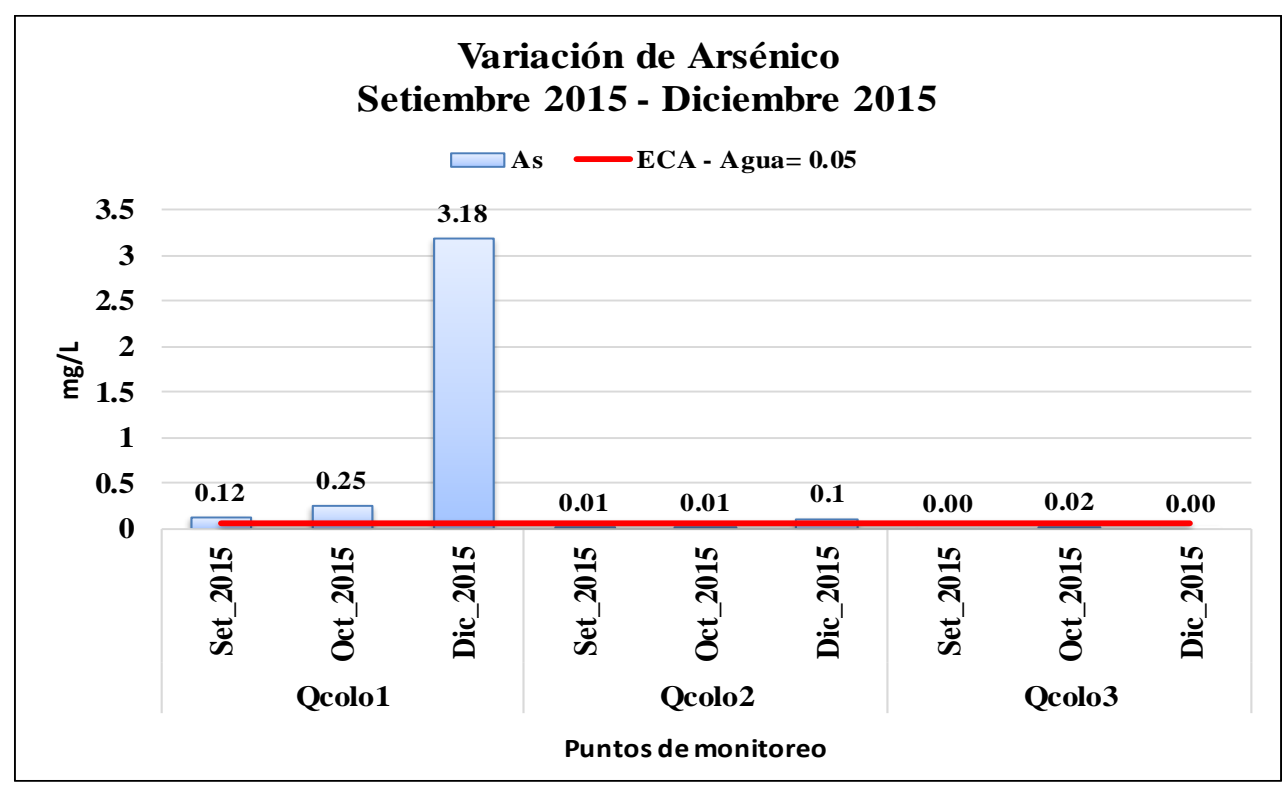

Figura 5. Variación de arsénico en la calidad del agua de la quebrada Colorada, setiembre - diciembre 2015. Según DS N002-2008 MINAN

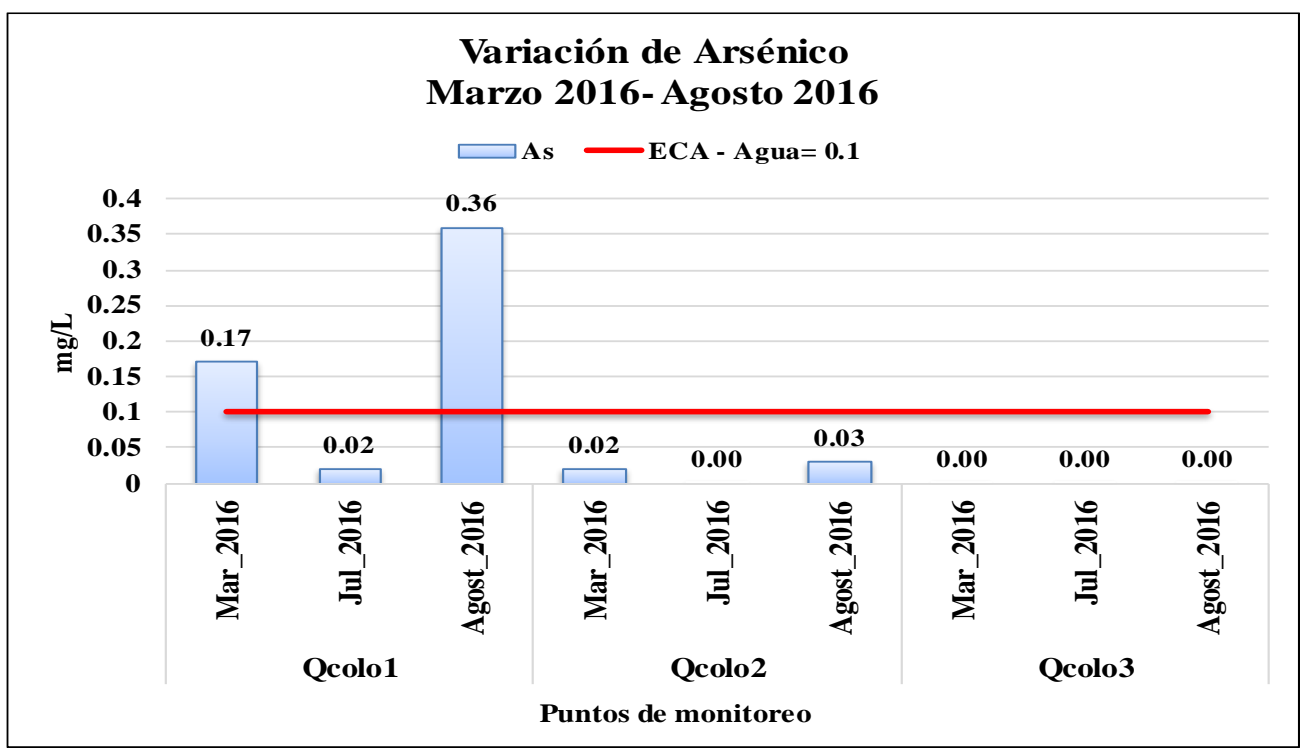

Figura 6. Variación de arsénico en la calidad del agua de la quebrada Colorada, marzo - agosto 2016. Según DS $\mathrm{N}^{\circ} 015-2015$ MINAN

\section{DISCUSIÓN}

En la quebrada colorada el $\mathrm{pH}$ acido se debe a la presencia de pasivos ambientales mineros ubicados en el paraje Sinchao, distrito de Chugur, registrando valores por debajo del límite inferior del rango establecido (6.5-8.5) del ECA-Agua para la categoría 3, el mismo que concuerda con el estudio 
realizado por Kirschbaum (2012) donde determinó el brusco descenso del pH y la solubilización inmediata de todos los elementos químicos presentes.

En la quebrada Colorada la conductividad eléctrica varió desde QColo1 hasta QColo3 observándose mayores valores en el punto de monitoreo QColo1, originado por la presencia de pasivos ambientales mineros. En la quebrada Colorada la presencia de calcio es porque hubo indicios de remediación con cal y observamos mayor concentración en el primer punto de monitoreo QColo1.

La presencia de aluminio, arsénico, cadmio, cobalto, cobre, hierro, manganeso, plomo y zinc es originado por la escorrentía que lavan los suelos de los pasivos ambientales mineros, cuyos valores registrados excedieron lo establecido en el ECA - Agua para la categoría 3, el que guarda concordancia con el estudio realizado por Kirschbaum (2012) donde determinó que, el aporte de metales está relacionado que los procesos de oxidación de actividades de minas, existiendo aún importantes volúmenes de material inalterado, por lo que se infiere que el potencial de generación de drenaje ácido es alto y la afectación al medio ambiente continuará en el tiempo en tanto no se tomen medidas de remediación y saneamiento en estos sitios.

En la quebrada Colorada se observó mayor concentración de metales aluminio, arsénico, cadmio, cobalto, cobre, hierro, manganeso, plomo y zinc en el punto de monitoreo QColo1, ubicado a $500 \mathrm{~m}$ de los pasivos ambientales mineros; el estudio realizado por Kirschbaum (2012) confirma esta premisa, en donde determinó que el aporte de los metales intensifican los procesos de oxidación y alteración de sulfuros que ocurren en los desechos mineros; Romero, Flores y Pacheco (2010) ratifica los resultados obtenidos en consideración a su investigación sobre la calidad del agua en el punto de Monitoreo P-205 es el segundo punto de la toma de muestra de aguas superficiales (M2), el cual se toma en las filtraciones de las aguas de la Cancha de Relave Polimetálico que va directamente a la cuenca del río Santa, el cual es el punto de monitoreo que presenta mayores concentraciones de arsénico (0.108 ppm), cadmio (0.024 ppm) y antimonio (0.01 ppm), en comparación con los demás puntos de monitoreo de aguas superficiales ".

Se ha realizado el análisis estadístico de las medias por periodos de muestreo en la quebrada Colorada identificando que, en los meses de diciembre 2015, julio y agosto del 2016 hubo mayor presencia de parámetros físicos - químicos que influenciaron en la variación de la calidad del agua en la quebrada Colorada, debido a la época de transición en (diciembre) 2015 y estiaje (julio y agosto) según el ciclo hidrológico histórico de la cuenca.

En los puntos de muestreo el ANAVA muestra diferencias significativas en el pH ( $\mathrm{p}=0.000<0.005)$, SST $(p=0.002<0.005)$, Cond $(p=0.000<0.005), \mathrm{Ca}(\mathrm{p}=0.000<0.005), \mathrm{Mg}(\mathrm{p}=0.000<0.005), \mathrm{Na}$ $(\mathrm{p}=0.000<0.005), \quad \mathrm{Al} \quad(\mathrm{p}=0.000<0.005), \quad \mathrm{Cd} \quad(\mathrm{p}=0.000<0.005), \quad \mathrm{Co} \quad(\mathrm{p}=0.000<0.005), \mathrm{Cu}$ $(\mathrm{p}=0.000<0.005), \mathrm{Fe} \quad(\mathrm{p}=0.000<0.005), \mathrm{Mn}(\mathrm{p}=0.000<0.005), \mathrm{Zn} \quad(\mathrm{p}=0.000<0.005)$ esta variación 
significativa se debe a la presencia de los pasivos ambientales mineros ubicados en el distrito de Chugur. Cabe precisar que en el análisis estadístico el parámetro Litio (Li) arrojo (p=0.001<0.005) siendo esta variación significativa y no influye en la calidad del agua comparado con el ECA-Agua para la categoría 3.

Sin embargo, los parámetros que no presentan diferencia significativa son los siguientes: DQO $(\mathrm{p}=0.571>0.005), \quad \mathrm{O} 2 \quad(\mathrm{p}=0.048>0.005), \quad \mathrm{K} \quad(\mathrm{p}=0.191>0.005), \quad \mathrm{Sb} \quad(\mathrm{p}=0.409>0.005), \quad$ As

$(\mathrm{p}=0.203>0.005), \quad$ Ba. $\quad(\mathrm{p}=0.421>0.005), \quad$ Be $\quad(\mathrm{p}=0.399>0.005), \quad \mathrm{B} \quad(\mathrm{p}=0.136>0.005), \quad \mathrm{Hg}$ $(\mathrm{p}=0.598>0.005), \mathrm{Pb}(\mathrm{p}=0.191>0.005)$.

Del análisis realizado con la prueba Tuckey se comprueba lo siguiente: La presencia del pH en el punto de monitoreo QCcolo3 es diferente comparado con el QColo1 y QColo2 debido a que en el recorrido a este punto existen afluentes de buena calidad del agua que aumentan el caudal y diluyen la concentración de sólidos suspendidos totales. Asimismo, se observa mayor valor del metal Na en el punto de monitoreo QColo3, esto se debe a la presencia de la diversidad de flora en este punto.

La presencia de SST, Cond, $\mathrm{Ca}, \mathrm{Mg}, \mathrm{Al}, \mathrm{Cd}, \mathrm{Co}, \mathrm{Cu}, \mathrm{Fe}, \mathrm{Li}, \mathrm{Mn}, \mathrm{Zn}$, en el punto de monitoreo QColo1 es diferente a los puntos de monitoreo QColo2 y QColo3, originado por la escorrentía de los pasivos ambientales mineros del paraje Sinchao (Socavones con agua y sin agua, planta en abandono, laguna acida (cleopatra), Cancha de relaves sin remediar, Desmonte de tierra con indicios de inicio de cierre, Tajo María Eugenia) ubicados a 500 metros aguas abajo.

\section{CONCLUSIONES}

Se identificaron catorce (14) pasivos ambientales mineros (Socavones con agua y sin agua, planta en abandono, laguna acida (cleopatra), Cancha de relaves sin remediar, desmonte de tierra con indicios de inicio de cierre, Tajo María Eugenia) existentes ubicados en el Paraje Sinchao, en el distrito de Chugur, provincia de Hualgayoc, que tienen influencia directa en la calidad del agua en la quebrada Colorada.

Con respecto a la calidad físico química del agua en la quebrada colorada se puede afirmar que el valor de $\mathrm{pH}$ es preponderantemente acido llegando a un valor de 2.90 asimismo los metales aluminio, arsénico, cadmio, cobalto, cobre, hierro, manganeso, plomo y zinc registraron valores que excedieron lo establecido en el ECA - Agua para la categoría 3.

El impacto de los pasivos ambientales mineros en la variación de la calidad del agua en la quebrada Colorada por medio del análisis de varianza se determinó que existe la presencia de pH, SST, Cond, 
$\mathrm{Ca}, \mathrm{Na}, \mathrm{Al}, \mathrm{CD}, \mathrm{Cu}, \mathrm{Fe}, \mathrm{Mn} \mathrm{Zn}$ con valores que difieren significativamente con los estándares de calidad del agua.

\section{REFERENCIAS BIBLIOGRÁFICAS}

Arango, M. (2011). Requerimientos para el diseño de una metodología que permita estimar el valor de pasivos ambientales mineros (Tesis de Maestría). Universidad Nacional de Colombia, Medellín, Colombia.

Arango, M. y Olaya, Y. (2012). Problemática de los pasivos ambientales mineros en Colombia. Revista Gestión y Ambiente. 15(3), 125-133.

Cedrón, M. (2013). Elaboración de criterios para la transformación de pasivos ambientales en activos socio - ambientales sostenibles (Tesis de Maestría). Pontificia Universidad Católica del Perú, Lima, Perú.

Culqui, L. y Culqui, M. (2015). Efecto antropogénico sobre la variación de la calidad del agua en la cuenca del rio Chancay - Lambayeque. (Tesis de Maestría). Universidad Nacional Pedro Ruiz Gallo, Lambayeque - Perú.

Decreto Supremo N $^{\circ}$ 059-2005-EM sobre reglamento de pasivos ambientales de la actividad minera. (2005). Recuperado de http://intranet2.minem.gob.pe/web/archivos/dgaam/publicaciones/compendio99/ds0592005.pdf

Fondo Nacional del Ambiente. (2007). Conferencia técnica: gestión de los pasivos ambientales mineros. Cajamarca, Perú.: Justo, J.

Instituto de Investigación y proyección sobre el ambiente natural y sociedad. (2015). Calidad del agua. Guatemala. Recuperado de http://www.infoiarna.org.gt/guateagua/subtemas/3/3_Calidad_del_agua.pdf

Instituto Nacional de Ecología y Cambio Climático. (2012). Calidad del agua. México. Recuperado de

http://www2.inecc.gob.mx/publicaciones/libros/612/calidad.pdf

Kirschbaum, A., Murray, J., Arnosio, M., Tonda, R. y Cacciabue, L. (2012). Pasivos ambientales mineros en el noroeste de Argentina: Aspectos mineralógicos, geoquímicos y consecuencias ambientales. Revista Mexicana de Ciencias Biológicas. 29(01), 248-264.

Ley $\mathrm{N}^{\circ} 28271$ sobre regulación de los pasivos ambientales de la actividad minera. (2004). Recuperado de 
http://www.tecnologiaslimpias.cl/peru/docs/LEYN_28271.pdf

Ley $\quad \mathrm{N}^{\circ} 28611$ sobre ley general del ambiente. (2005). Recuperado de http://cdam.minam.gob.pe/novedades/leygeneralambiente2.pdf

Manahan, S. E. (2007). Introducción a la química ambiental. México. Editorial Reverte.

Oblasser, A. \& Chaparro, E. (2008). Estudio comparativo de la gestión de los pasivos ambientales mineros en Bolivia, Chile, Perú y Estados Unidos. Santiago de Chile, Chile.: Publicaciones de las Naciones Unidas.

Pinto, H. (2013). Los pasivos mineros ambientales y los conflictos sociales en Hualgayoc. Revista de Investigación de la UNMSM. 03(30). Recuperado de http://revistasinvestigacion.unmsm.edu.pe/index.php/sociales/article/view/8033

Rebolledo, E. (2011). Informe de valoración de pasivos socio-ambientales vinculada a la actividad minera aurífera ilegal en el norte de esmeraldas. Reporte técnico. Recuperado de https://www.researchgate.net/publication/280052412

Resolución Jefatural $N^{\circ}$ 010-2016-ANA sobre protocolo nacional para el monitoreo de la calidad de los recursos hídricos. (2016). CUT 135807-2015. Recuperado de http://www.ana.gob.pe/sites/default/files/normatividad/files/r.j._010-2016-ana_0.pdf

Romero, A., Flores, S., y Pacheco, W. (2010). Estudio de la calidad del agua de la cuenca del rio Santa. Revista del Instituto de Investigación FIGMMG. 13(25). Recuperado de http://sisbib.unmsm.edu.pe/bibvirtual/publicaciones/geologia/v13_n25/pdf/a09v13n25.pdf

Russy, D. y Martinez-Alier, J. (2003). Los pasivos ambientales. Revista de Ciencias Sociales. 01(15), 123-131. 